\title{
Signal Processing in Telecommunications with Forward Correction of Errors
}

\author{
Juliy Boiko ${ }^{1}$, Oleksander Eromenko² \\ ${ }^{1}$ Department of Telecommunications and Radio Engineering, Khmelnytsky National University, \\ 29016 Institutskay a 11, Khmelnytsky, Ukraine \\ ${ }^{2}$ Department of Physics and Electrical Engineering, Khmelnytsky National University \\ 29016 Institutskay a 11, Khmelnytsky, Ukraine
}

\begin{tabular}{|c|c|}
\hline Article Info & ABSTRACT \\
\hline Article history: & \multirow{9}{*}{$\begin{array}{l}\text { The development of mechanisms of increase efficiency of frequency-shift } \\
\text { key ing signals processing in telecommunications using algorithms of noise } \\
\text { immunity channel coding in obstacle effect conditions is held in the article. } \\
\text { The synthesis of the frequency-shift keying signal processing unit accounting } \\
\text { intersymbol communication which is inherent for such signals with } \\
\text { continuous phase is held. The conditions of the compromise implementation } \\
\text { in the telecommunication information transmission channel with frequency } \\
\text { shift keying and error correction coding for setting the optimal encoding rate } \\
\text { in the range of the bandwidth of the information transmission system are } \\
\text { explored. Linear cyclic codes Bose-Chaudhuri-Hocquenghem (BCH) are } \\
\text { used for studying. By means of Matlab the article focuses on the definition of } \\
\text { energetic benefit compared to uncoded system in case of equality of the } \\
\text { bandwidth of the information transmission system with coding and without } \\
\text { coding. }\end{array}$} \\
\hline Received Apr 2, 2018 & \\
\hline Revised Apr 22, 2018 & \\
\hline Accepted Jun 14, 2018 & \\
\hline Keywords: & \\
\hline Modulation & \\
\hline Telecommunications & \\
\hline Signal-code construction & \\
\hline Optimum receiver & \\
\hline
\end{tabular}

Copyright $\odot 2018$ Institute of Advanced Engineering and Science. All rights reserved.

\section{Corresponding Author:}

Juliy Boiko,

Department of Telecommunications and Radio Engineering,

Khmelnytsky National University,

29016 Institutskaya 11, Khmelnytsky, Ukraine.

Email: boiko_julius@ukr.net

\section{INTRODUCTION}

The digital frequency shift keying (FSK) signals are widespread used in modern telecommunications, in particular standards for cellular communication of GSM, TETRAPOL, DECT, radio modems, audiocontrol systems and some satellite channels of information transmission. Gauss frequency shift keying (GMSK) has some important benefits: the compact range and the possibility of coherent detection [1-4].

The main problem is to find the effective of signal-code construction (SCC), methods of generating signals of the frequency shift keying and the synthesis and the development of optimal device for receiving and processing frequency shift keying signal depending on the duration of the transition process in the filtering circuit and setting a minimum limit hypotheses level to minimize distortion of bit parcels. The solution of this problem will allow to improve the quality of digital telecommunication channels $[5,6]$.

Discussed issues in the article and the problem statement for the research can be described as follows: the introduction to the scheme of formation of block coding signals leads to the bandwidth increase of information flow in contrast with the case when the modulation of information signals is exclusively used in the information transmission system [1]. Therefore, it is important to establish the best compromise between modulation and coding for a given bandwidth in the case of information transmission in telecommunication systems. 
The goal of the article lies in the study of the conditions of compromise implementation in the telecommunication channel of information transmission with frequency modulation and error correction encoding for the setting the optimal encoding rate in the range of the bandwidth of the information transmission system. In addition, the task of the definition of energetic benefit compared to uncoded system in case of the bandwidth equality of the information transmission system with coding and without coding is needed to be explored. For research in the work the linear cyclic codes Bose-Chaudhuri-Hocquenghem $(\mathrm{BCH})$ with interleaving will be used. The main advantage of such a signal-code construction building is that the group errors are reformed in single ones and a discrete channel without memory is formed for the codec [7-9] The coder usage increases the number of bits for transmission in the information transfer system. In this case, the length of bit sequences must be reduced to ensure a constant rate of information transmission. The decrease of the duration of bit sequences will increase the bandwidth of the information transmission system. Such circumstances indicate that the generating signals device in coding system should contain a filter with a smaller bandwidth for comparing when the encoding is missing, to ensure a fixed width of total throughput [1]. Therefore, it is necessary to propose an approach to implement the generating signals devices which will provide a balance between coded and uncoded information transmission systems. A synthesis of receiving and processing device of such signals regarding the duration of the transition process in the circuit of filtration and installation the minimum edge layer of hypotheses with the aim to minimize the distortion of bit sendings is suggested in this paper.

As well as the article is devoted to the approaches for ensuring the efficiency of SCC usage and the features of realization of the bits interleaving devices in telecommunications.

\section{RESEARCH METHOD}

While designing the telecommunication systems a problem of envisaging a highly reliable transmission of digital information by channels with noise arises. The methods of protecting data from errors is used to solve this problem and are based on the use of theory and specific algorithms for decoding on the basis of noise immunity codes. Most of these algorithms are heuristic. The noise immunity codes allow to get an energetic benefit coding (EBC), which characterizes the degree of possible reduction of energy transfer when encoding compared with the absence of coding, if the requirements for the reliability of transmission in both cases are the same. The specific meaning of the energetic (and economical) effect which was got with the help of coding EBC is contained in a substantial reduction of transmitter power, saving bandwidth, increasing the communication range and the ability to work at very high noise of channel. In addition, it can provide a significant increase in data transmission rate and its accuracy, as well as many other useful technological benefits under equal circumstances coding. Keeping in mind the pointed out benefits of coding, the greater focus is placed on the problem of EBC increasing in the design of telecommunication devices and the development of simple and efficient decoding algorithms is an important and first-priority problem.

The importance of solving the urgent problems of the theory and practice of using telecommunication devices makes an important scientific and technical challenge, which is in the develop of effective signal-code constructions that provide a high quality of information transfer in a relatively narrow band of frequencies occupied by the signal, while envisaging the most simple implementation, which confirms the urgency of the tasks solved in the article.

\subsection{Features of Signals Formation}

Carry on the synthesis of the signal processing unit the frequency-shift keying considering intersymbol connection which is inherent in such signals with continuous phase.

The general scheme of frequency-shift signal formation is shown in Figure 1 [1], [4], [9].

$$
U(t)=U_{m} \cos \left(2 \pi f_{0} t+\psi_{t}\right)
$$

where $U_{m}$ - the amplitude of the signal; $2 \pi f_{0}=\omega_{0}$ - carrier frequency; $\psi_{t}$ - the phase of the signal.

$$
\psi_{t}=S_{k} \pi h / T\left(t-(k-1)-T_{0}\right)+\pi h \sum S_{j}+\psi_{0}
$$

where $h=2 \pi \Delta f_{d} T_{0} ; \Delta f_{d}$ - frequency deviation; $S_{k} \in\{ \pm 1\}$ - information signs; $\psi_{0}$ - a random value of the initial phase. 


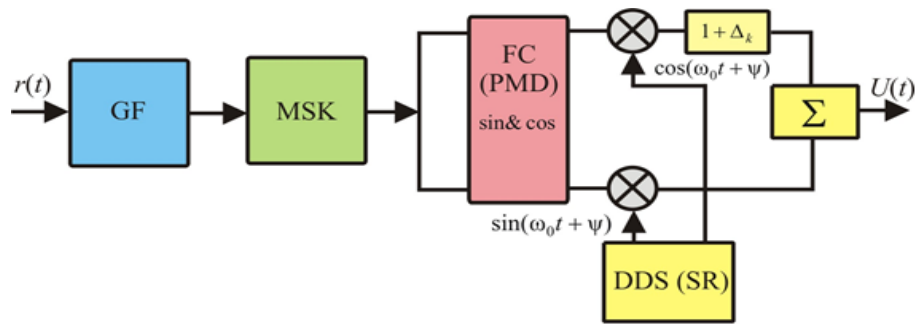

Figure 1. Functional diagram of the formation of frequency-shift signal: GF - gauss filter; MSK - frequencyshift keying; FC (PMD) sin\&cos - functional converter (a permanent memory device); $\Delta_{k}$ - the coefficient of ambiguity; DDS (SR) - digital synthesiser (synthtsiser reference); $\sum$ - adder

The signal with the frequency-shift can be analytically presented in the following way: there is another variant of signals formation scheme of the frequency-shift keying, using a cascade connection of gauss filter and voltage controlled generator (VCG). However, in this case, the implementation of generating devices and formation of frequency-shift signals should be realized considering the following circumstances: while using synthesisers in the generating signals circuits of frequency-shift keying one should regard that the data arrays which represent a sequence of ones and zeros have a spectral opinion which is applied to almost constant values of the signals. In this case, most modern synthesisers will be insensitive to such lowfrequency signals (typical synthesiser has a frequency characteristic of low frequencies filter). In this case, it is advisable to use quadrature scheme Figure 2 and a diagram with thermocompensation quartz generators, which are excluded from the loopback for frequency. Thermocompensated generators (TCG) allow to obtain the frequency stability from $\pm 0.5 \mathrm{ppm}$ to $\pm 10 \mathrm{ppm}$. The type of output of such generators is - CMOS and clipped Sine. The scheme of this generator is shown in Figure 2. In the scheme one part of the signal goes to the VCG and the second part is used to modulate TCQG. Thus, the quartz generator is excluded from the feedback loop. So, TCQG can be modulated by a low frequency signal and its output signal is effectively added to the signal which modulates VCG in the synthesiser. The combined signal will have a spectral opinion which is applied to a continuous signal.

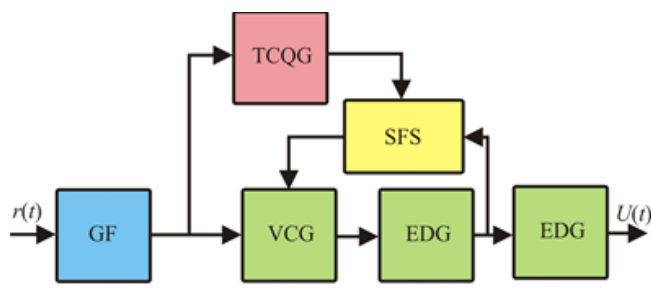

Figure 2. The scheme of formation the frequency-shift keying signal: TCQG - the thermocompensated quartz generator; SFS - a synthesiser of frequency shift; VCG - a voltage controlled generator; EDG - an external drive generator

Synthesis of quadrature modulator can be performed using the Laurent decomposition for the complex envelope GMSK which is represented as a linear combination of a sequence of pulses [10].

$$
\begin{aligned}
& U(t)=U_{m} \sum_{n=-\infty}^{\infty}\left[b_{2 n} C_{0}\left(t-2 n T_{0}\right)-b_{2 n+1} b_{2 n} b_{2 n-1} C_{1}\left(t-2 n T_{0}-T_{0}\right)\right]+ \\
& j U_{m} \sum_{n=-\infty}^{\infty}\left[b_{2 n+1} C_{0}\left(t-2 n T_{0}-T_{0}\right)-b_{2 n} b_{2 n-1} b_{2 n-2} C_{1}\left(t-2 n T_{0}\right)\right]
\end{aligned}
$$

Figure 3 shows the first three generated pulses $C_{0}, C_{1}, C_{2}$ obtained by means of Matlab. In particular from the obtained graphs for $h=0,5, B \cdot T_{0}=0,25$ one can determine that the first two pulses of 
decomposition have the sufficient amount, while the third pulse $C_{2}$ is barely noticeable. In fact, one pulse $C_{0}$ is sufficient for linearity approximation of GMSK modulation.

Both components (3) are used for a signal generate and their signal trajectories are shown in

Figure 4. Figure 5 presents the eye-diagram for the case $B \cdot T_{0}=0,25$. To minimize the intersymbol interference the equalizer based on the Wiener filter was added to the stock of abovementioned schemes of formation with the code conversion [1].

In general, the schemes of signals formation with frequency shift keying which are shown in Figure 1 and 2 can be supplemented by a scheme of the previous differential encoding. The possible options for this configuration of the generating device and signals formation are presented in Figure 6.
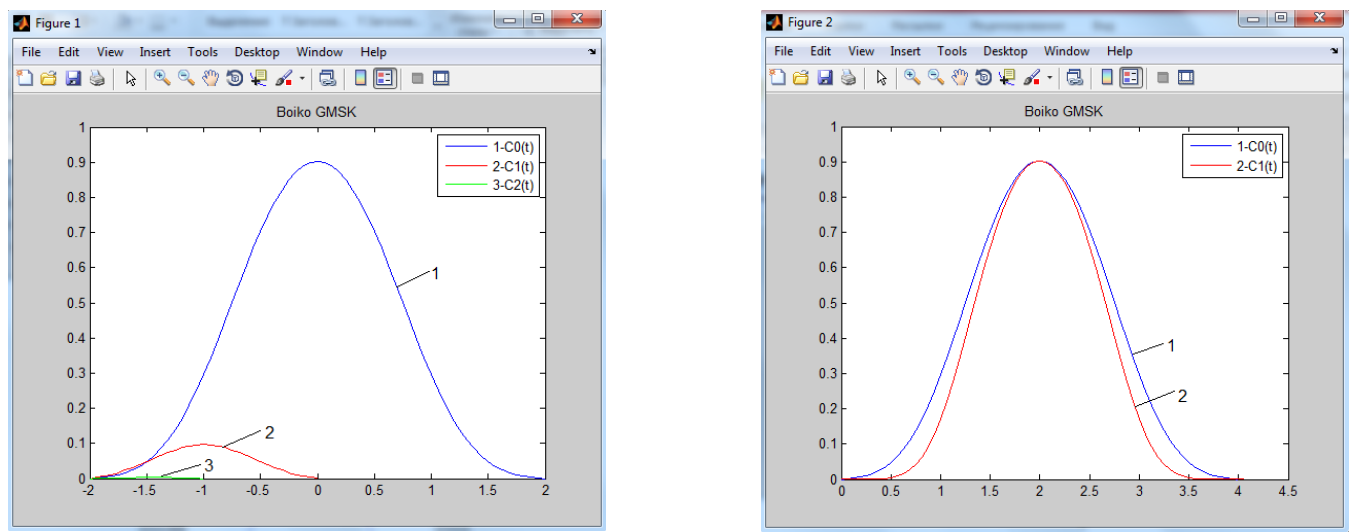

Figure 3. The pulses of the Laurent decomposition for the complex envelope of GMSK: $1-C_{0} ; 2-C_{1} ; 3-C_{2}$
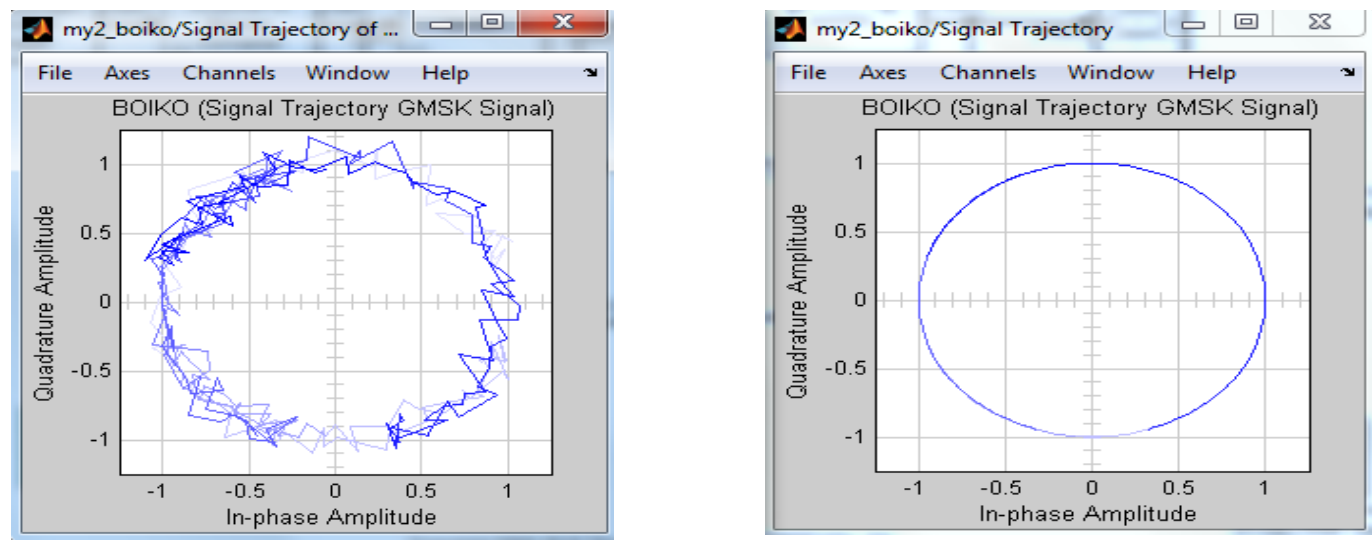

Figure 4. GMSK signal trajectory for the amplitude component (3), $B \cdot T_{0}=0,25$

\subsection{The Method of Optimization of the Device Signal Processing}

Use the above approaches to the formation of frequency-shift keying signals, and carry out the synthesis of the device for receiving and processing such signals.

The impact on the receiving part is analytically presented by the following:

$$
\bar{\eta}=\bar{\eta}(t)=U(Y, X, t)+\bar{n}(t)
$$

where $\bar{\eta}(t) \quad$ - the vector implementations of the processed signals; $U_{t}(Y, X)=U(Y, X, t)=u_{1}(Y, X, t), u_{2}(Y, X, t), u_{3}(Y, X, t), \ldots u_{n}(Y, X, t)$ - a set of known functions of 
time and stochastic processes; $\bar{n}(t)=\left(n_{1}(t), n_{2}(t), n_{3}(t), \ldots, n_{n}(t)\right)$ - vector of white Gauss noise in telecommunications transmission channel with statistical characteristics, $\langle\bar{n}(t)\rangle=0$ $\left\langle\bar{n}(t) u^{-\mathrm{T}}(t+\tau)\right\rangle=1 / 2 N \delta(\tau)$ and $N$ - is the matrix of the spectral noise density; $\delta(\tau)$ - Dirac function; T - the designation of the transpose operation.

The initial ratio for building models of the signal processing unit via aposteriori the probability distribution of a discrete process is presented in the following way:

$$
P\left(U_{1}^{k+1} \mid \eta^{-k+1}\right)=C_{k+1} P\left(U_{k+1} \mid U_{k}\right) F\left(U_{k+1}\right) P\left(U_{1}^{k} \mid \eta_{1}^{-k}\right)
$$

where $C_{k+1}$ - is the constant of normalization; $P\left(U_{k+1} \mid U_{k}\right)$ - is the transition matrix.

$$
\left.F\left(U_{k+1}\right)=e^{\left[\int_{k T_{0}}^{(k+1) T_{0}} f\left(U_{k+1}, t\right) d t\right.}\right]
$$
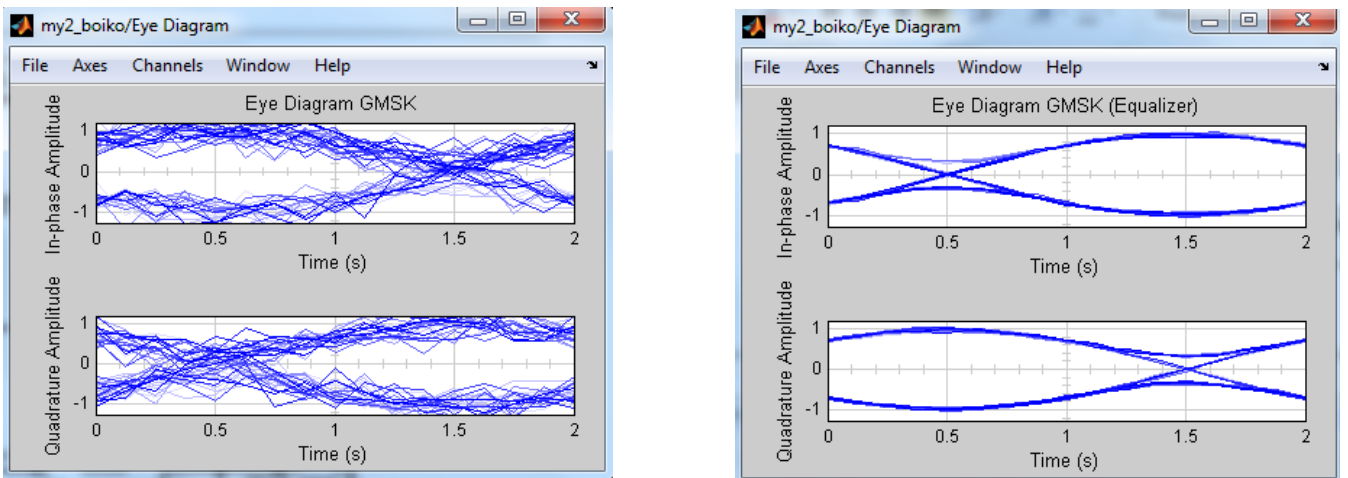

Figure 5. The eye-diagrams of GMSK $B \cdot T_{0}=0,25$ to as sess the effect of intersymbol interference compensation
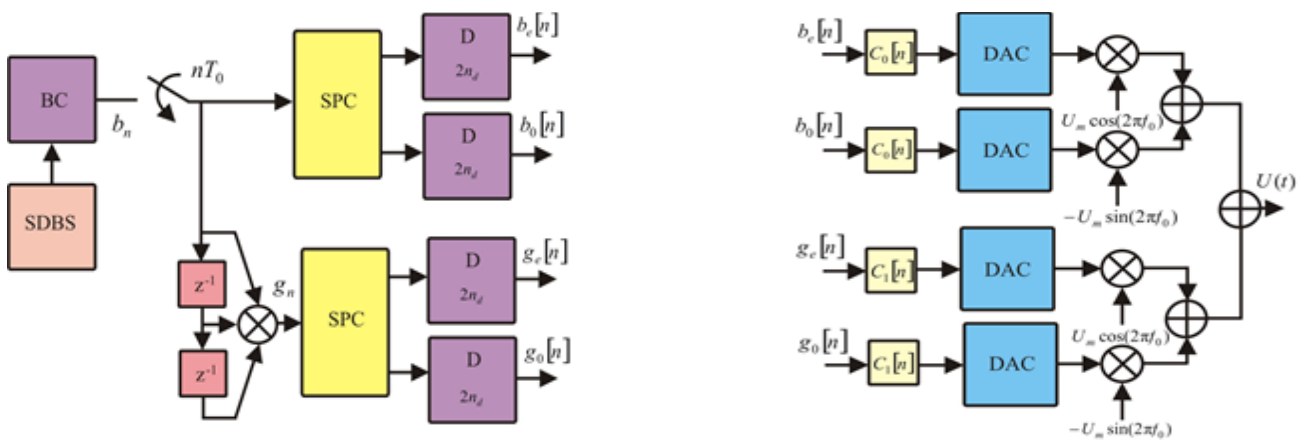

Figure 6. The circuit implementation of the quadrature trans mitter with frequency shift keying: SDBS source data of a bit stream; BC - bit converter; D - the device of sampling rate; DAC - digital-to-analog converter; SPC - serial-parallel converter

$$
f\left(U_{k+1,}, t\right)=-\left[\bar{\eta}_{t}-U_{t}\left(U_{k+1}\right)\right]^{\mathrm{T}} N^{-1}\left[\bar{\eta}_{t}-U_{t}\left(U_{k+1}\right)\right],
$$


likelihood function; $T_{0}$ - is the clock interval of the transmission of digital signals; $U_{k}$ - the value of the discrete process on $\mathrm{k}$ the clock interval $\left((k-1) T_{0} \leq t<k T_{0}\right) ; U_{1}^{k}$ - is the sequence of values of a discrete signal: $U_{1}, U_{2} \ldots U_{k}$.

In accordance with the mathematical model (4) the input action is presented in such a way:

$$
\bar{\eta}_{t}=\sqrt{\frac{2 E_{b}}{T_{0}}} \cos \left(2 \pi f_{0} t+\psi_{t}\right)+n_{t}
$$

where $E_{b}$ - the energy of the signal during one information bit; $\psi_{t}=\psi(t)$ - the phase of the signal; $2 \pi f_{0}=\omega_{0}$ - carrier frequency; $n_{t}$ - gauss white noise with spectral density $N_{0} / 2$.

The signals in the quadrature channels are presented by the following expression:

$$
\left.\begin{array}{l}
\eta_{1 t}^{\prime}=\sin \psi_{t}+\sigma_{u} \zeta_{1 t} \\
\eta_{2 t}^{\prime}=\cos \psi_{t}+\sigma_{u} \zeta_{2 t}
\end{array}\right\}
$$

where $\zeta_{1 t}$ and $\zeta_{2 t}$ - are correlated in time and uncontrolled between themselves gauss processes with unit variance, and root-mean-square value of noise is described by the expression:

$$
\sigma_{u}=\sqrt{\left(m T_{0} / 4 h_{0}^{2}\right)} .
$$

To describe the phase-frequency shift keying signal the following expression is used:

$$
\psi(t)=S_{k} \frac{\pi h}{T_{0}}\left(t-(k-1) T_{0}\right)+\pi h \sum_{j=1}^{k-1} S_{j}+\psi_{0}, \quad(k-1) T_{0} \leq t \leq k T_{0}
$$

The likelihood function will be analytically presented as follows, according to expression (6):

$$
\left.F\left(U_{k+1}\right)=e^{\left[4 / N_{0} \int_{k T_{0}}^{(k+1) T_{0}} \eta_{1 t} U_{1 t} d t+4 / N_{0}\right.} \int_{k T_{0}}^{(k+1) T_{0}} \eta_{2 t} U_{2 t} d t\right],
$$

where

$$
U_{1 t}=U_{1}(t)=\sqrt{\frac{E_{b}}{2 T_{0}}} \sin \psi_{t} \quad U_{2 t}=U_{2}(t)=\sqrt{\frac{E_{b}}{2 T_{0}}} \sin \cos \psi_{t}
$$

Using the expression (5) and (12) and one can get:

$$
\lg P\left(U_{1}^{k+1} \mid \bar{\eta}_{1}^{k+1}\right)=\lg C_{k+1}+\lg P\left(U_{k+1} \mid U_{k}\right)+\frac{4}{N_{0}} \int_{k T_{0}}^{(k+1) T_{0}} \eta_{1 t} U_{1 t} d t+\frac{4}{N_{0}} \int_{k T_{0}}^{(k+1) T_{0}} \eta_{2 t} U_{2 t} d t+\lg P\left(U_{1}^{k} \mid \bar{\eta}_{1}^{k}\right)
$$

In the expression (13)

$$
P\left(U_{k+1} \mid U_{k}\right) \text { is used as a requirement component, and the vector }
$$

means a set of quadrature components $\eta_{1 t}, \eta_{2 t}$.

Consider the process of algorithmic presentation of the proposed scheme of optimal signals processing with frequency-shift keying in more details. As the source expression (13) for the simulation of noise immunity will be adopted the one. It should be clearly noted that a strictly optimal algorithm will provide its exact implementation but it is almost impossible due to the progressive increase of the number of possible combinations of bits and time. However, it is possible the mutual bits influence on account of the scenarios correlation is limited, as well as their full amount of which is seen in an every moment of time. Use this condition to simplify building of the lightweight algorithm with the possibility of its practical implementation. 
In view of the fact that the rule of limiting the number of possible hypotheses (combinations of bits) is contained that sets the maximum number of hypotheses which is discarded half with the smaller weights $P\left(U_{k+1} \mid U_{k}\right)$ after their weighing according to formula (13). Each of the hypotheses, which is remained for the next measure is complemented by zero and one, and two new hypotheses are being formed. The reference signals $U_{X r 1}$ and $U_{X r 2}$ are generated for each hypothesis to implement the formula (12). As the initial value, the last k-th bit estimate isn't used and the bit offset to the depth of the given scenario, i.e. $(k-i)$ bit is used. In this case, the probability of correct recognition increases with the increasing of i. Greater focus should be placed on the implementation of such an algorithm which should provide a clear understanding of the fact that on the one hand the theoretical improvement is limited by the duration of the transition process in the filtering scheme, and the other hand is an artificial limit of the number of hypotheses on account of a lack of computing power, which may lead to distortion of the bit with a considerable delay.

In the case of receiving a frequency-shift signal, the task of the receiving path is to recover the phase trajectory of the transmitted signal which for an interval of time $n T<t<(n+1) T$ can be represented as follows:

$$
\psi\left(t, \phi_{n}\right)=2 \pi h \sum_{j=n-L+1}^{n} \varphi_{j} \cdot q(t-j T)+\pi h \sum_{j<n-L+1}^{n} \varphi_{j}=2 \pi h \sum_{j=n-L+1}^{n} \varphi_{j} \cdot[q(t-j T)-1 / 2]+\pi h \sum_{j<n}^{n} \varphi_{j}
$$

where $h=2 \pi \Delta f_{d} T_{0} ; \Delta f_{d}$ - is the frequency deviation.

Then the first term will represent as the product of the intersymbol interference and evaluate the phase state:

$$
\Theta_{n-1}=\pi h \sum_{j<n}^{n} \varphi_{j}, \quad \varphi_{n}=\frac{\left(\Theta_{n}-\Theta_{n-1}\right)}{h \pi}
$$

So, by comparing the phase states on the border of the clock interval, symbol is transmitted at this interval can be defined (in the case $h=1 / 2$ have $\Theta \in\{0, \pi / 2, \pi, 3 \pi / 2\}$ ), and the value $\Theta_{n}$ can be estimated by the signs of the quadrature components.

The integrated scheme of the optimal receiver is shown in Figure 7.

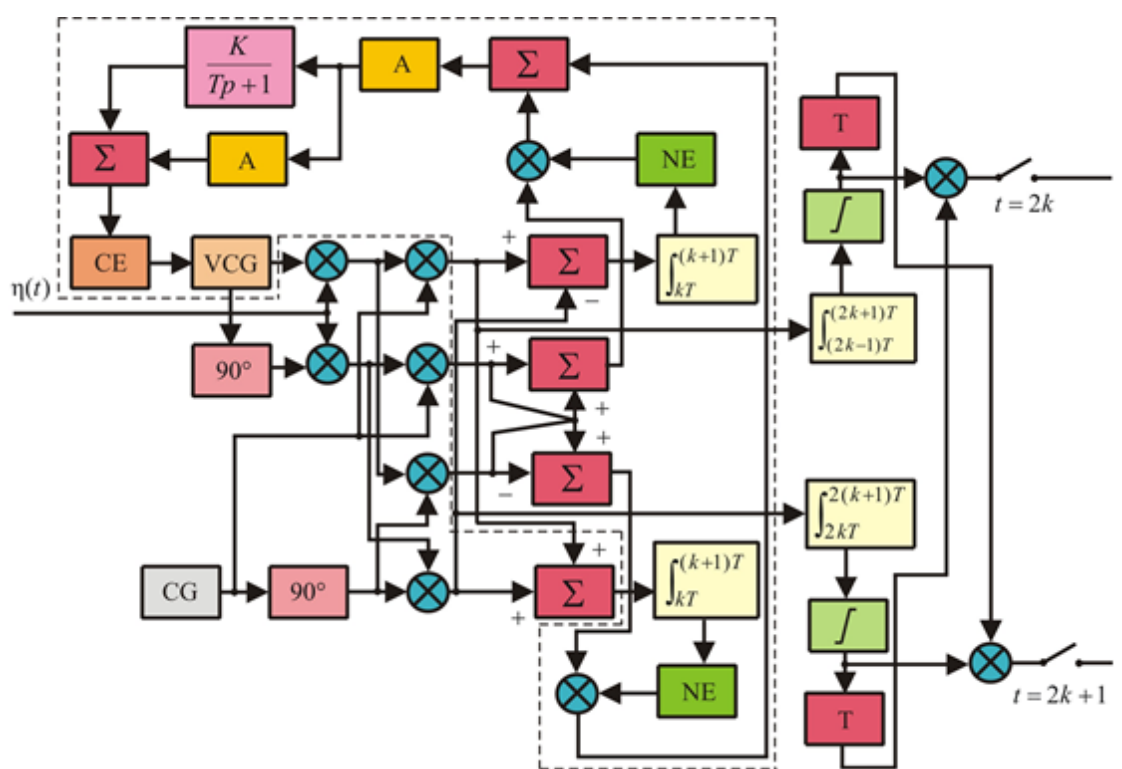

CG - clock generator; $\mathrm{CE}$ - controlled element; 90 - phase shifting device; NE - nonlinear element; VCG - controlled generat or

Figure 7. Structural scheme of the optimal receiver FSK signal with a circle of synchronization 


\section{RESULTS AND DISCUSSION}

The research results of noise immunity signals of frequency shift keying indicate that the best features has the coherent reception of FSK signals. Thus, at the BER level of 10-5 the energy gain of MSK (coherent acceptance) before DQPSK is $1.5 \mathrm{~dB}$, and before non-coherent reception -4 dB [9], [11-13].

The results of simulation of telecommunication channel with noise immunity coding and frequency shift keying carried out in [9], [14], [15] allow to establish that the greatest noise immunity has the SCC based on convolutional codes, and the energy gain reaches almost to $5.7 \mathrm{~dB}$ and $\mathrm{BCH}$-codes (selected for study) energy gain reaches nearly $2.6 \mathrm{~dB}$ for level $\mathrm{BER}=10-5$. Proposed in the paper sheet-oriented decisions regarding to the formation and processing of FSK signals in combination with BCH codes let get the energy gain of up to $4.2 \mathrm{~dB}$ in the case of providing an efficient balance between Gauss filter bandwidth on the transmission side and the $\mathrm{BCH}$ code rate. In order to modulate it's appropriate to involve bigger bandwidth when the bandwidth system is small. In the opposite case, when bandwidth system is large, it's advisable to attract a large bandwidth for encoding.

Table 1 shows experimental results of evaluating noise immunity of the SCC: MSK+BCH for different configuration codes, for the purpose of determining the EBC [16], [17].

Figure 8 and Figure 9 show the graphs of noise immunity for the studied $\mathrm{BCH}$ codes with the aim of determining the probability of symbol error [7], [14]:

$$
p_{s} \leq \frac{1}{n} \sum_{i=t+1}^{n}(i+t) C_{n}^{i} p_{0}^{i}\left(1-p_{0}\right)^{n-i}
$$

where $n_{-}$is the number of code symbols in the block; $t^{t}$ - is the number of corrected errors (correction capacity of the code); $p_{0}$ - is the average probability of error in a bit information.

Table 1. The results of the noise immunity evaluation of SCC on the basis of BCH codes

\begin{tabular}{cccc}
\hline \multicolumn{2}{c}{ Encoding rate, the SCC parameters $(\mathrm{n}, \mathrm{k})$} & Level BER & $\mathrm{EBC}, \mathrm{dB}$ \\
\hline \multirow{4}{*}{$1 / 2$} & $(7,4)$ & & 0,4 \\
& $(15,7)$ & & 1 \\
& $(31,16)$ & $10-5$ & 1,7 \\
& $(127,30)$ & & 2,6 \\
$(255,123)$ & & 3,5 \\
& $(511,256)$ & 3,7 \\
& $(1023,513)$ & & 4,1 \\
& $(15,5)$ & & 1,2 \\
& $(15,11)$ & & 1,3 \\
& $(31,11)$ & & 2,1 \\
& $(31,21)$ & $10-5$ & 2,12 \\
& $(63,18)$ & & 2,7 \\
& $(63,45)$ & & 3,1 \\
& $(127,43)$ & & 3,5 \\
\hline
\end{tabular}

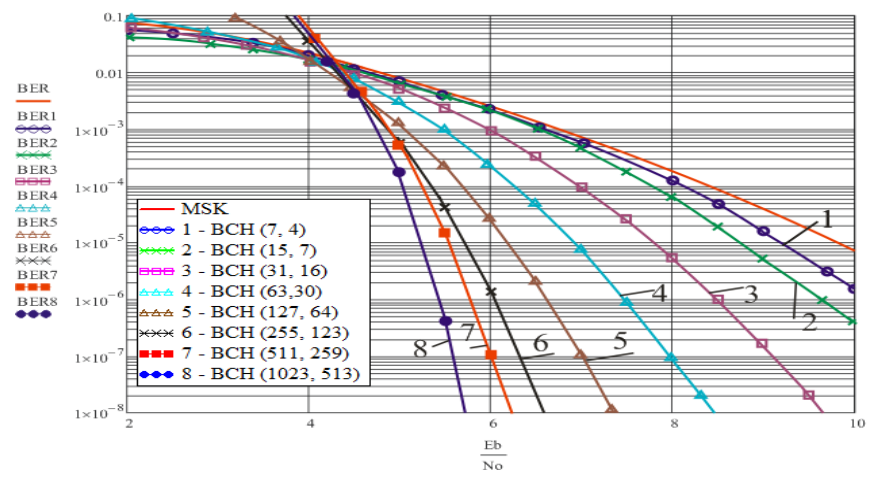

Figure 8. Graphs of noise immunity for the SCC on the bas is of $\mathrm{BCH}$ codes with different configurations $(\mathrm{R}=1 / 2): 1-(7,4) ; 2-(15,7) ; 3-(31,16) ; 4-(63,30) ; 5-(127,64) ; 6$ - $(255,123) ; 7$ - $(511,259) ; 8-$ (1023, 513); MSK for lack of coding 


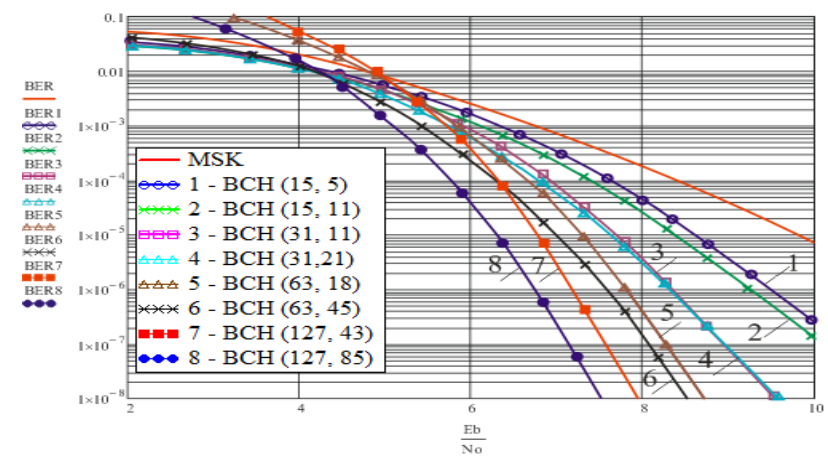

Figure 9. Graphs of noise immunity for the SCC on the basis of $\mathrm{BCH}$ codes with different configurations $(\mathrm{R}=1 / 3,2 / 3): 1-(15,5) ; 2-(15,11) ; 3-(31,11) ; 4-(31,21) ; 5-(63,18) ; 6-(63,45) ; 7-(127,43) ; 8-$ (127, 85); MSK for lack of coding

Figure 10 shows the result of the study of compromise in choosing the optimal balance between coding and modulation depending on the bandwidth of the telecommunication system with frequency -shift keying. Conducted researches for the proposed SCC with the aim to determine the maximum efficiency are systematized in Table 2.

Table 2. The parameters SCC GMSK+BCH

\begin{tabular}{lcc}
\hline \multirow{2}{*}{ Bandwidth } & $\begin{array}{c}\text { Parameters of the SCC }(\mathrm{GMSK}+\mathrm{BCH}-\mathrm{BT} 0,(\mathrm{n}, \mathrm{k}, \mathrm{t})) \\
\mathrm{n}=255\end{array}$ \\
\hline 1,18 & $0,194(255,223,4)$ & $0,170(127,106,3)$ \\
1,28 & $0,224(255,215,5)$ & $0,215(127,106,3)$ \\
1,63 & $0,327(255,187,9)$ & $0,406(127,99,4)$ \\
1,80 & $0,448(255,187,9)$ & $0,441(127,95,2)$ \\
1,90 & $0,451(255,179,10)$ & $0,410(127,85,6)$ \\
1,96 & $0,442(255,171,11)$ & $0,442(127,85,6)$ \\
2,02 & $0,457(255,171,11)$ & $0,456(127,85,6)$ \\
2,05 & $0,458(255,171,11)$ & $0,458(127,85,6)$ \\
\hline
\end{tabular}

The obtained results can be offered to discuss questions of increase of productivity of functioning of modems and telecommunications codecs with frequency shift-keying.

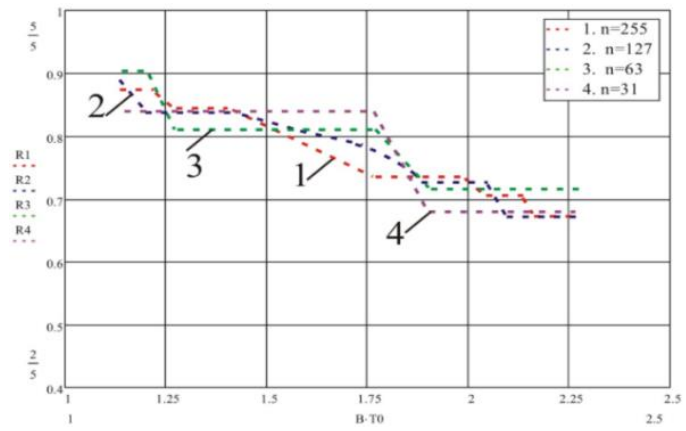

Figure 10. Dependencies of the code rate R due to come up to the maximum productivity the SCC codes of based on $\mathrm{BCH}$ codes from the total bandwidth telecommunication systems for different sizes of code blocks $n$

\section{CONCLUSION}

The article presents the description of the synthesis process of optimal processing signals device with frequency-shift keying for constructing of noise immunity signal-code design of telecommunication systems. The obtained results can be used in the discussion of the following statements: using synthesiser in 
circuits generating signals frequency-shift keying considering that the data arrays representing a sequence of ones and zeros have a spectral opinion which is applied to almost constant values of the signals. In this case, it is advisable to use the quadrature scheme and the scheme with thermocompensation quartz generators which are excluded from the loopback frequency. In the case of signals transmission of frequency-shift keying occurs the phase uncertainty $\pm \pi$, which leads to the effect of "reverse work" of demodulator. In addition, most methods of the synchronization and carrier recovery are conducive to coming out the phase uncertainty. Synthesis of quadrature modulator frequency-shift signal can be performed using the Laurent decomposition for the complex envelope for the GMSK which is a linear combination of a sequence of pulses. The conducted researches allow to establish: to minimize the intersymbol interference to the containment of abovementioned schemes of formation with the code conversion it is reasonable to add the equalizer based on the Wiener filter. Performed description of the synthesis the receiving and processing device the frequency-shift signal depends on the duration of the transition process in the filtering scheme and setting a minimum limit level of hypotheses to minimize distortion of bit sendings. For a given bandwidth of the telecommunication system using $\mathrm{BCH}$ codes with Gauss frequency shift keying, the selection of the optimal code configuration depends on a number of factors, which in particular include the length of the code block and the measure throughput system. The code rate which helps to achieve the best production, is approximately $2 / 3$. The study indicates that the energy efficiency of SCC on the basis of BCH codes with decreasing the code rate decreases, and the maximum energy gain reaches $4.2 \mathrm{~dB}$.

It is reasonable to focus the further researches on the establishment of usage the efficiency in telecommunication channels of non-binary codes built in fields GF(25), GF(26), GF(28), GF(27) for low values ratio of the signal/noise for different signal-code constructs. It needs to be determined the optimal values of the encoding rates and the optimal balance between modulation and coding for these SCC.

\section{REFERENCES}

[1] Rappaport TS. "Wireless communications: principles and practice". New Jersey: Prentice Hall, 1996: 641.

[2] De Buda R. "Coherent demodulation of frequency-shift keying with low deviation ratio". IEEE Transactions on Communications. 1972; 20(3): 429-435.

[3] The Anh Nguyen Dinh, Huy Le Xuan, Tuan Anh Vu, Duong Bach Gia. "A Status Data Transmitting System for Vessel Monitoring". International Journal of Electrical and Computer Engineering. 2018; 2(8), 917-925.

[4] Mohamed Amine Jhaidri, Christophe Laot, Alain Thomas. "Nonlinear analy sis of GMSK carrier phase recovery loop". Proceedings of the 2016 International Symposium on Signal, Image, Video and Communications (ISIVC), Tunis, Tunisia. 2016: 230-235.

[5] Mohamed Syed Ali. "Reconfigurable Modulation Scheme for Communication System". Indonesian Journal of Electrical Engineering and Computer Science. 2018; 9(3): 624-628.

[6] Linda M, Nur A, Iswahyudi H. "Performance of Coded Modulation in Multicarrier CDMA System". Indonesian Journal of Electrical Engineering and Computer Science. 2016; 1(3): 583-589.

[7] Roth RM. "Long cy clic codes over $\mathrm{GF}(4)$ and $\mathrm{GF}(8)$ better than $\mathrm{BCH}$ codes in the high-rate region". IEEE Trans. Inform. Theory. 2017;63(1): 150-158.

[8] Deevya Indoonundon, Tulsi Pawan Fowdur, Sunjiv Soyjaudah. "A Concealment Aware UEP scheme for H.264 using RS Codes". Indonesian Journal of Electrical Engineering and Computer Science. 2017; 6(3): 671-678.

[9] Eko Marpanaji, Bambang Riyanto, Armein Z.R. Langi, Adit Kurniawan. "Pengukuran unjuk kerja modulasi gmsk pada software-defined radio platform". TELKOMNIKA (Telecommunication Computing Electronics and Control). 2007; 2(5), 73-84.

[10] Laurent P. "Exact and Approximate Construction of Digital Phase Modulations by Superposition of Amplitude Modulated Pulses (AMP)". IEEE Transactions on Communications. 1986; 34(2): 150-160.

[11] Pratima M, Soni MK. OFDM PAPR "Reduction Using Recurring SLM with Cyclic and Linear Block Codes Schemes". Indonesian Journal of Electrical Engineering and Computer Science. 2015; 16(1): 1-6.

[12] Lydia Sari, Antonius Aditya. "Raptor Code for Energy-Efficient Wireless Body Area Network Data Transmission". TELKOMNIKA (Telecommunication Computing Electronics and Control). 2015; 1(13), 277-283.

[13] Boiko J, Kovtun I, Petrashchuk S. "Productivity of telecommunication systems with modified signal-code constructions". Proceedings of the 2017 4th International IEEE Scientific-Practical Conference Problems of Infocommunications. Science and Technology (PIC S\&T), Ukraine, 2017; 173-178.

[14] Pankaj Kr. Das. "Codes Detecting and Correcting Solid Burst Errors”. Bulletin of Electrical Engineering and Informatics. 2012; 3 (1), 225-232.

[15] Giordano AA, Levesque AH. "Digital Communications BER Performance in AWGN (FSK and MSK). Indianapolis": Wiley Telecom. 2015: 416.

[16] Boiko YuM, Boryachok RO. "Improving effectiveness for processing signals in data transmission channels with phase manipulation". Proceedings of the 2013 23rd International IEEE Crimean Conference Microwave and Telecommunication Technology (CriMiCo), Ukraine, 2013; 262-263.

[17] Shynkaruk O, Boiko J, Eromenko O. "Measurements of the energy gain in the modified circuit signal processing unit". Proceedings of the 2016 13th International IEEE Conference on Modern Problems of Radio Engineering. Telecommunications and Computer Science (TCSET), Ukraine, 2016; 582-584. 